\title{
Self-Similarity Sparse Representation for Image Restoration
}

\author{
D Khalandar Basha, T Venkateswarlu
}

\begin{abstract}
Image restoration aims to restore an image from a degraded image. The degradation may occur during image acquisition or image transmission. Image degradation lowers the quality of the image. In this paper additive Gaussian noise is considered for degrading the original image. For restoring the image from degraded image the proposed method used both local and non-local similarity patterns. The restoration problem is modeled with regression model. Two regularization terms are considered for representing prior image information. One regularization term is for local patterns and other is for non-local similarity patterns. The additive local regularization term is used to restore the edges. The non-local regularization term works best for local smoothness and edge information will be lost. The proposed algorithm took a clean image of size $256 \times 256$ and added with Gaussian noise with different levels of noise levels. A self-adaptive dictionary is trained for a particular window of image with local and non-local patterns and stacked to three dimensional matrix. The patch size considered for training the dictionary is 10x10. For restoring each patch it searches best atoms form the trained dictionary. The efficiency of the algorithm is estimated by parameters mean square error, root mean square error, PSNR and FSIM. The algorithm is also tested for different images like cameraman, house, Barbara, Lena and parrot. The proposed algorithm is tested with conventional algorithms. .
\end{abstract}

Index Terms-Image restoration, Sparse representation, Gaussian noise, Dictionary learning, self-similarity.

\section{INTRODUCTION}

In Image processing the noise present in the image is one of the predominant factor causing low quality of the image. The noise introduced during image capture has to eliminate for further processing of the image in later stages. The process of removal of noise from the image to reconstruct the image like original image is called image restoration. The lesser the difference between original image and reconstructed image, the more removal of noise. The efficiency of the algorithm depends on less displacement between reconstructed image and original image.

Let the original image is $\mathrm{x}$, and $\mathrm{y}$ is the degraded image, represented as

$$
y=H x+\eta
$$

Where $\eta$ is the noise added to the transformed image. The noise can be additive or multiplicative, is always unknown quantity. The $\mathrm{H}$ is degradation function. The function $\mathrm{H}$ defines the functionality like image restoration, image

Revised Version Manuscript Received on 16 September, 2019.

D Khalandar Basha, Asst. Professor, Department of ECE, Institute of Aeronautical Engineering, Dundigal, Hyderabad, Telangana, India.

Research Scholar, Department of ECE SVU College of Engineering, Tirupathi, Andhrapradesh, India.(Email: bashavlsi@gmail.com)

Dr. T Venkateswarlu, Professor, Department of ECE SVU College of Engineering, Sri Venkateswara University, Tirupathi, Andhrapradesh, India.

(Email: warlu57@gmail.com) deblurring or compressive sensing. If $\mathrm{H}$ is a diagonal matrix with elements as either 0 or 1 in diagonal then the equ (1) is image restoration. As $\eta$ is unknown the error is given by

$$
e=H x-y
$$

The (2) won't give exact difference between original image and reconstructed image as noise is unknown. To overcome this ill-posed image restoration, image prior information is needed and added for regularizing and the solution for (1) is minimizing problem given by

$$
\arg \min _{x} \frac{1}{2}\|H x-y\|_{2}^{2}+\lambda \varphi(x)
$$

arg min argument is $l_{2}$ norm of mean square error and $\varphi(x)$ is regularization term specifies image prior information and

$\lambda>0$ is Lagrange multiplier called regularization parameter. There are many optimization algorithms proposed for above problem (3) like total variation [1-2], Group based Sparse representation [3] etc. Sparsity property of natural image used for regularization in image denoising. In this method each patch is reconstructed by dictionary, trained from natural images.

The efficiency of image restoration depends on the dictionary and patch. Non local means NLM [4] used mainly nonlocal regularization term uses non local self-similarity in the images. GSR groups the patches to restore the image [5, 6]. In this paper for regularization term used both local and non-local similarity pattern in patches to learn dictionary.

\section{CONVENTIONAL METHODS}

In image restoration using constrained optimization in conventional methods constraints are applied using Lagrange multiplier and solution are obtained by gradient methods [7, 8]. In these methods with assumption that the images are locally smooth except at edges of the image. The design of regularization term gives the effectiveness of restoration problem. The dictionary set consists of patches of each pattern in one column. If the dictionaries trained well then the restoration problem performance is increased as dictionary learning is employed to images. K-SVD method [9] optimizes sparse approximation in the learned dictionary. This method gives how to train the dictionary by using non local similarity in the image for a given patch.

In an image the repetition of the pattern exists example like the most of the background pattern, textures and structures are spread in most regions. The non-local similarity method is designed by assumption that the pattern of the image may be repeated. For a pattern in a patch may be present in same 
type structure in window or any non-local patches. The sparsity enhancement done by BM3D [10] techniques, the patches are stacked into 3D instead of 2D. The non-local regularization is effective compared to local regularization method [11]. In non-locally centralized sparse representation (NCSR) [12].

\section{SELF-SIMILARITY SPARSE REPRESENTATION}

In this paper sparse representation of natural images modeled by using both self-similarities between local patches and non-local patches. In non-local self-similarity the edges information are losing. For restoring the edge information local similarity are added while learning the dictionary. The method consists of patch creation, sparse representation, learning dictionary.

As in (3), the regularization term is composed of two properties local regularization term $\phi(x)$ and non-local regularization term $\varphi(\mathrm{x})$. The Lagrange multiplier for local and non-local regularization terms are $\mu_{1}, \mu_{2}$. The ill posed problem (3) represented with both regularization terms as

$$
\arg \min _{x} \frac{1}{2}|| H x-y||_{2}^{2}+\mu_{1} \phi(x)+\mu_{2} \varphi(\mathrm{x})
$$

Equ (4) is image restoration problem using both local and non-local similarities. Local similarity term gives the difference between pixels to pixels. This can be estimated by applying gradient to the image which will restore the edge information. The gradient is calculated at any location of pixel is

$$
x(i, j)-x(i-1, j)+x(i, j)-x(i, j-1)
$$

Equ (5) is used as local regularization term for local similarity to restoring the image. The non-local similarity depict the local smoothness in non-local regions. On great success of non-local smoothness in BM3D method for image restoration applications [13]. This paper also used the non-local similarity property for image restoration.

Consider an image $x \in R^{N}$ construct the patches of size 10x10 non-overlapping. Then search for $\mathrm{n}$ best patches similar to original image window. The best patches are stacked into a dictionary.

For each patch of image find sparse vector. This sparse vector contains few coefficients with non-zero entries. Repeat this process for all patches of the image. This process inserting into equ (4) as

$$
\arg \min _{x} \frac{1}{2}\|H D \propto-y\|_{2}^{2}+\mu_{1} \phi(x)+\mu_{2} \varphi(x)
$$

The success of image restoration depends on the dictionary D. Fetching the sparse coefficients from the dictionary used techniques like MOD [14], PCA and SVD [15]. Equ (6) contains three constraints namely observation constrain, local constrain and non-local constrain. Solving of above equ leads to image restoration problem. Solution for (6) can be obtained by using Split Bregman Iteration algorithm [16]. This SBI converts unconstraint problem into constraint minimization problem by Bregman iteration. The SBI iteration is given in Table1.
Table 1: Split Bregman Iteraion (SBI)

Step 1: Set $\mathrm{i}=0, \mu>0, \mathrm{~b} 0=0, \mathrm{u} 0=0, \mathrm{v} 0=0$

Step 2: $u^{i+1}=\arg \min _{u} f(u)+\frac{\mu}{2}\left\|u-G v^{i}-b^{i}\right\|_{2}^{2}$

Step 3: $v^{i+1}=\arg \min _{v} g(v)+\frac{\mu}{2}\left\|u^{i+1}-G u-b^{i}\right\|_{2}^{2}$

Step 4: $b^{i+1}=b^{i}-\left(u^{i+1}-G v^{i+1}\right)$;

Step 5: $i=i+1$;

Step 6: stop if criteria is reached else repeat step 2

The SBI algorithm is used to solve unconstrained problem by converting into constrain problem.

The flow of the algorithm is represented in table 2

Step1: Read original image and add Gaussian noise Step 2: Solve local regularization term for the equation

$$
\arg \min _{x}=\frac{1}{2}\|y-x\|_{2}^{2}+\mu \varphi(x)
$$

Where $\phi(x)$ is a local regularization parameter. It is first derivative (gradient).

Step 3: Solve non local regularization term for the equation

$$
\arg \min _{x}=\frac{1}{2}\|y-x\|_{2}^{2}+\mu \varphi(x)
$$

Where $\varphi(x)$ is a non-local regularization parameter. It is sparse approximation.

Step 4: Check for stopping criteria If stopping criteria is reached stop the iterative process else go to step2.

After executing the above algorithm restored image is obtained. The efficiency of the algorithm is estimated by the parameters MSE, RMSE, PSNR and FSIM.

The non-local regularization process begins with creating patches of size $10 \times 10$ then search for best similar patches from the dictionary. The best pattern patches are stacked to dictionary. Then apply the transform. For sparse representation pick non-zero transform coefficients. Then reconstruct the image using the sparse coefficients.

\section{RESULTS}

The performance of the proposed methods are analyzed comparing with few existing methods. The proposed method is implemented in Matlab2016. The images considered for testing of the proposed method are shown in Fig.1. The images are added with Gaussian noise. The noise is added with different noise levels $0.1,0 \mathrm{n} .2,0.3,0.4$, and 0.5 . The performance of the algorithm is estimated by the parameters mean square error, root mean square error, PSNR and FSIM. The images are considered of size $256 \times 256$. The patch size considered are $10 \times 10$ to creating dictionary. The values of Lagrange multiplier considered are 0.0017 and 0.0145 . The number of iterations are 30 . The results are tabulated.

The MSE parameter gives dissimilarity between restored image and original image. The MSE values is error parameter between those two images. Table 1 gives the comparison of 

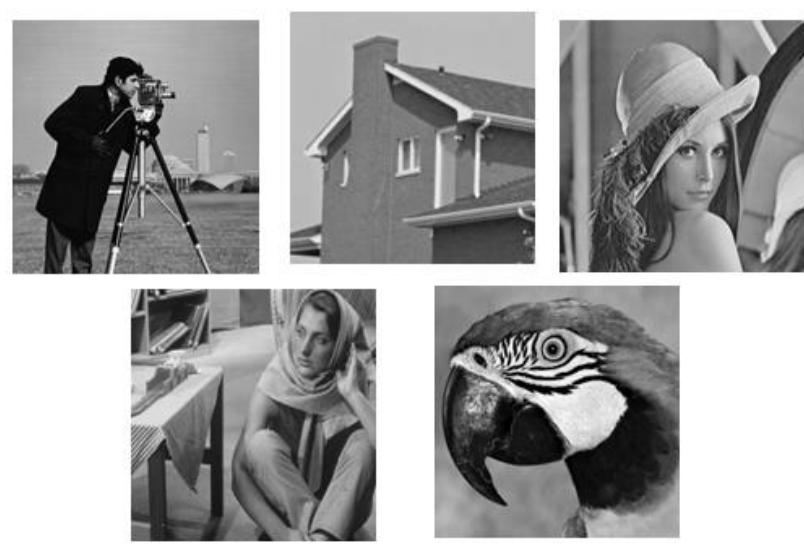

Fig 1: Test Images

Table 1: MSE measurement for different Gaussian noise levels.

\begin{tabular}{|l|l|l|l|l|l|}
\hline $\begin{array}{l}\text { Noise } \\
\text { Level }\end{array}$ & 0.1 & 0.2 & 0.3 & 0.4 & 0.5 \\
\hline $\begin{array}{l}\text { Camera } \\
\text { man }\end{array}$ & 0.088 & 0.0880 & 0.0887 & 0.0893 & 0.0901 \\
\hline House & 0.1766 & 0.1779 & 0.1800 & 0.183 & 0.1883 \\
\hline Lena & 0.3609 & 0.3627 & 0.3660 & 0.3705 & 0.3759 \\
\hline Barbara & 0.1644 & 0.1646 & 0.1687 & 0.1619 & 0.1657 \\
\hline Parrot & 0.0749 & 0.0751 & 0.0754 & 0.0761 & 0.0772 \\
\hline
\end{tabular}

Table 2: RMSE measurement for different Gaussian noise levels.

\begin{tabular}{|l|l|l|l|l|l|}
\hline $\begin{array}{l}\text { Noise } \\
\text { Level }\end{array}$ & 0.1 & 0.2 & 0.3 & 0.4 & 0.5 \\
\hline $\begin{array}{l}\text { Camera } \\
\text { man }\end{array}$ & 0.938 & 0.9382 & 0.942 & 0.944 & 0.949 \\
\hline House & 0.4203 & 0.4218 & 0.4242 & 0.4279 & 0.4339 \\
\hline Lena & 0.6001 & 0.6022 & 0.6050 & 0.6087 & 0.6131 \\
\hline Barbara & 1.2825 & 1.2832 & 1.2840 & 1.2853 & 1.2871 \\
\hline Parrot & 0.8658 & 0.8664 & 0.8685 & 0.8722 & 0.8784 \\
\hline
\end{tabular}

MSE values of different images with various Gaussian noise levels. Lesser the MSE the more resembles restored image to the original image. Table 2 gives the RMSE value, it is square.

Table 3: PSNR measurement for different Gaussian noise levels.

\begin{tabular}{|l|l|l|l|l|l|}
\hline $\begin{array}{l}\text { Noise } \\
\text { Level }\end{array}$ & 0.1 & 0.2 & 0.3 & 0.4 & 0.5 \\
\hline $\begin{array}{l}\text { Camera } \\
\text { man }\end{array}$ & 28.68 & 28.68 & 28.65 & 28.62 & 28.58 \\
\hline House & 35.660 & 35.628 & 35.578 & 35.504 & 35.382 \\
\hline Lena & 32.557 & 32.535 & 32.496 & 32.442 & 32.381 \\
\hline Barbara & 25.97 & 25.96 & 25.959 & 25.951 & 25.939 \\
\hline Parrot & 29.382 & 29.376 & 29.355 & 29.318 & 29.257 \\
\hline
\end{tabular}

Table 4: FSIM measurement for different Gaussian noise levels.

\begin{tabular}{|l|l|l|l|l|l|}
\hline $\begin{array}{l}\text { Noise } \\
\text { Level }\end{array}$ & 0.1 & 0.2 & 0.3 & 0.4 & 0.5 \\
\hline $\begin{array}{l}\text { Camera } \\
\text { man }\end{array}$ & 0.927 & 0.9268 & 0.926 & 0.9255 & 0.9242 \\
\hline House & 0.9543 & 0.9542 & 0.9539 & 0.9537 & 0.9532 \\
\hline Lena & 0.9595 & 0.9592 & 0.9588 & 0.9581 & 0.9571 \\
\hline Barbara & 0.9801 & 0.9801 & 0.9800 & 0.9798 & 0.9795 \\
\hline Parrot & 0.9446 & 0.9463 & 0.9459 & 0.9450 & 0.9437 \\
\hline
\end{tabular}

PSNR is Peak signal to noise ratio. It is also dissimilarity measurement parameter. The value of PSNR should be more. PSNR is inversely proportional to log of MSE 


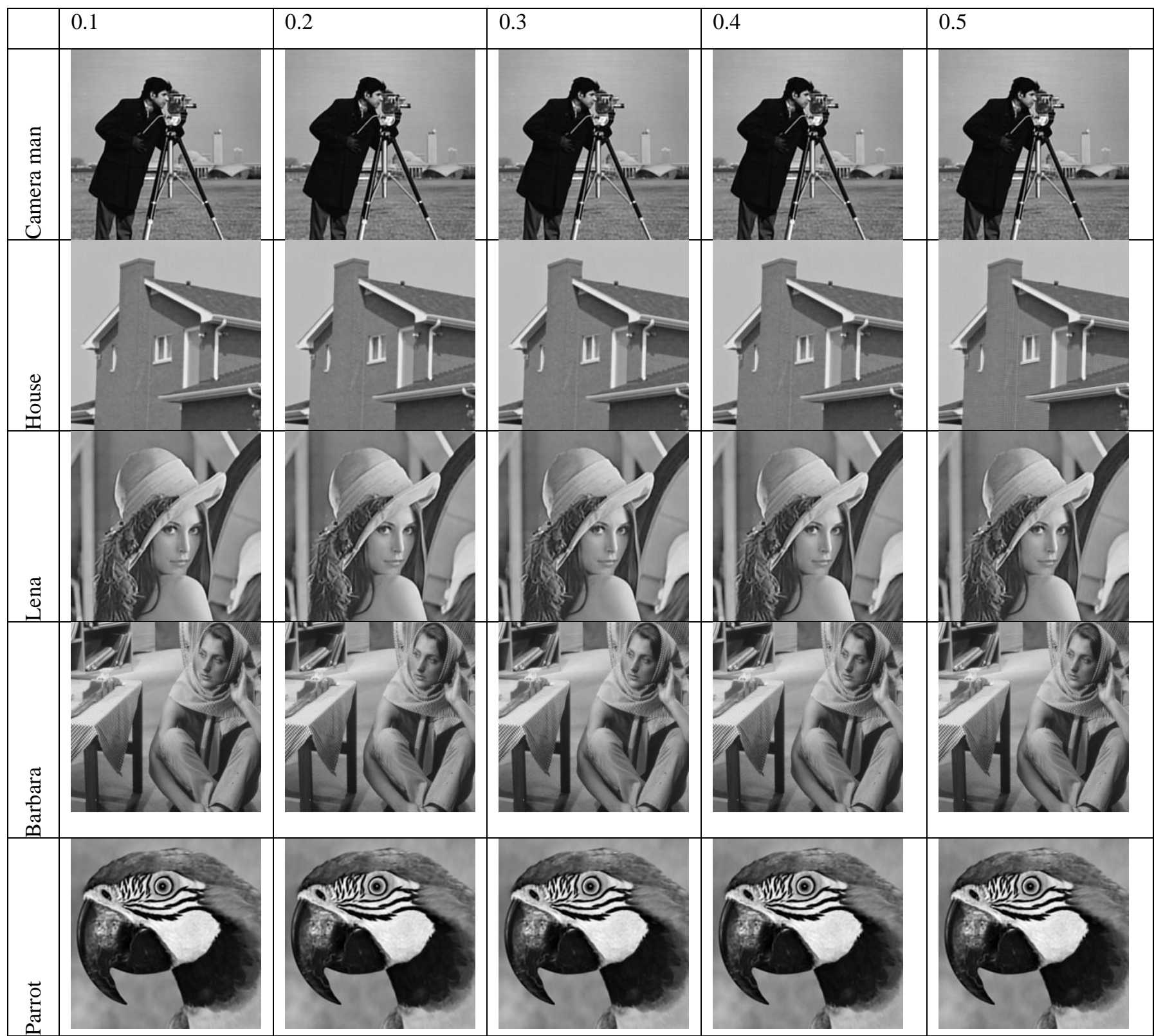

Fig 3: Restored images of Cameraman, House, Lena, Barbara and Parrot which are degraded by Gaussian noise levels of $0.1,0.2,0.3,0.4$ and 0.5

\section{CONCLUSION}

The aim of the image restoration is to remove the noise and the efficiency of the image restoration depends on how much closer the reconstructed image with the original image. The paper proposes self-similarity based sparse representation. The proposed algorithm is applied to normal images like Cameraman, Lena, House, Barbara and Parrot with added Gaussian noise of different noise levels of $0.1,0.2,0.3,0.4$ and 0.5 . The dictionary is constructed with patches of size 10x10. For image restoration both local and non-local similarities are used. Local similarity regularization term restored the edge information whereas the non-local similarity regularization term for restoration smoothness of the patch in image window. The performance of the image is analyzed by estimating the parameters like MSE, RMSE, PSNR and FSIM. The performance of the proposed algorithm is compared with the methods like SALSA [15], NCSR [15], TVMM [9] for PSNR and FSIM values. The proposed algorithm gave better results. In this paper performance of the algorithm tested for Gaussian noise future work extend for other noises like salt-pepper and speckle noises.

\section{REFERENCES}

1. L. Rudin, S. Osher, and E. Fatemi, "Nonlinear total variation based noiseremoval algorithms," Phys. D, Nonlinear Phenomena, vol. 60, nos. 1-4,pp. 259-268, Nov. 1992.

2. A. Chambolle, "An algorithm for total variation minimization and applications," J. Math. Imag. Vis., vol. 20, nos. 1-2, pp. 89-97, Jan./Mar. 2004.

3. J. Zhang, D. Zhao, and W. Gao, "Group-Based Sparse Representation for Image Restoration," IEEE transaction on image processing, vol. 23, No. 8, pp. 3336-3351, August 2014.

4. A. Buades, B. Coll, and J. M. Morel, "A non-local

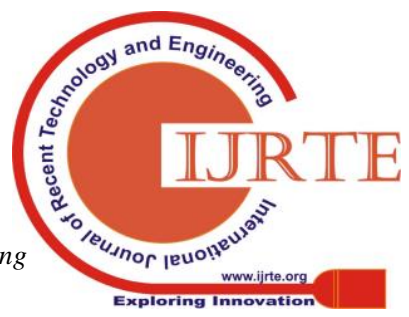


algorithm for image denoising," in Proc. Int. Conf. CVPR, Jun. 2005, pp. 60-65.

5. H. Ji, C. Liu, Z. Shen, and Y. Xu, "Robust video denoising using low rank matrix completion," in Proc. IEEE Conf. CVPR, Jun. 2010, pp. 1791-1798.

6. H. Ji, S. Huang, Z. Shen, and Y. Xu, "Robust video restoration by joint sparse and low rank matrix approximation," SIAM J. Imag. Sci.,vol.4, no. 4, pp. 1122-1142, 2011.

7. J. Bioucas-Dias, M. Figueiredo, and J. Oliveira, "Total-variation image decon-volution: A majorizationminimization approach," in Proc. IEEE Int. Conf. Acoust., Speech, Signal Process., Toulouse, France, pp. 861864, May 2006.

8. X. Zhang, M. Burger, X. Bresson, and S. Osher, "Bregmanized nonlocal regularization for deconvolution and sparse reconstruction," SIAM J. Imag. Sci.,vol. 3, no. 3, pp. 253-276, 2010.

9. M. Aharon, Michael Elad, and A. Bruckstein. K-SVD: An Algorithm for Designing Overcomplete Dictionaries for Sparse Representation. IEEE Transactions on image processing, pages 9-12, 2005 . http://dx.doi.org/10.1109/TSP

10. Marc Lebrun, An Analysis and Implementation of the BM3D Image Denoising Method, Image Processing On Line, 2 (2012), pp. 175-213. https://doi.org/10.5201/ipol.2012.1-bm3d

11. M. Jung, X. Bresson, T. F. Chan, and L. A. Vese, "Nonlocal Mumford-Shah regularizers for color image restoration," IEEE Trans. Image Process., vol. 20, no. 6 , pp. 1583-1598, Jun. 2011.

12. W. Dong, L. Zhang, G. Shi, and X. Wu, "Image deblurring and superresolution by adaptive sparse domain selection and adaptive regularization," IEEE Trans. Image Process., vol. 20, no. 7, pp. 1838-1857, Jul. 2011.

13. K. Dabov, A. Foi, V. Kat kovnik, and K. Egiazarian, "Image denoising by sparse 3D transform-domain collaborative filtering," IEEE Trans. on Image Process., vol. 16, no. 8, pp. 2080-2095, Aug. 2007.

14. K. Engan, S. O. Aase, and J. H. Hakon-Husoy, "Method of optimal directions for frame design," in Proc. IEEE Int. Conf. Acoust., Speech, Signal Process., vol. 5. Mar. 1999, pp. 2443-2446.

15. K. Engan, S. O. Aase, and J. H. Hakon-Husoy, "Method of optimal directions for frame design," in Proc. IEEE Int. Conf. Acoust., Speech, Signal Process., vol. 5. Mar. 1999, pp. 2443-2446.

16. T. Goldstein and S. Osher, "The split Bregman algorithm for L1 regularized problems," SIAM J. Imaging Sci., vol. 2, pp. 323-343, 2009. 